\title{
Ce que m'ont appris les Roughriders de la Saskatchewan au sujet de la pharmacie
}

\author{
par Jason Howorko
}

$\mathrm{D}$ ans la ligue canadienne de football, c'est l'équipe des Roughriders de la Saskatchewan qui est ma préférée. En novembre dernier, j'ai vu mon équipe perdre la Coupe Grey aux dépens des Alouettes de Montréal, dans ce que je décrirais comme l'une des parties les plus enlevantes qui m’ait été donné de voir.

Les Roughriders ont perdu par un seul point au tout dernier jeu. À peine quelques moments avant, les joueurs et leurs milliers de partisans croyaient bien avoir remporté la victoire. Malheureusement, à cause d'une pénalité aux Roughriders pour un trop grand nombre de joueurs sur le terrain, les Alouettes ont eu une chance supplémentaire pour tirer le but gagnant... et ils ont su tabler sur cette chance.

En repensant à tous ces événements des Roughriders, je me suis rendu compte qu'on pouvait dresser plusieurs parallèles avec notre profession.

Voir grand: Chaque année, nous, les partisans de la Saskatchewan (connus sous le nom de "Rider Nation ») gardons notre optimisme, croyons à notre équipe et la soutenons. Peu importe si elle joue mal, nous continuons à croire qu'un jour nous remporterons la Coupe Grey. Il n'y a pas si longtemps, l'idée du pharmacien prescripteur semblait impossible, mais aujourd'hui, l'évolution du rôle du pharmacien, comprenant le droit de prescrire, se fait sentir partout au pays.

Vision et leadership: Une vision à long terme (illustrée pour notre profession par le projet SCPH 2015 et le Plan directeur pour la pharmacie) est tout aussi importante pour la pharmacie que le sont le coaching et le livre de jeux pour les Roughriders.

Travail d'équipe : Le quart-arrière d'une équipe de football a besoin d'excellents joueurs de soutien afin d'atteindre les objectifs de l'équipe. Dans les soins de santé, chacun de nous joue un rôle unique et utile, et en travaillant en équipe nous pouvons avoir une influence considérable sur les soins aux patients.

Communication: Dans le football, il est important de savoir ce qui se passe, à la fois en dehors du terrain et sur le terrain. Dans la pratique de la pharmacie, l'information et une bonne communication sont essentielles à la prestation de soins adéquats. La mauvaise communication coûte cher.

Responsabilité: L'automne dernier, l'entraineur des Roughriders a d'emblée admis qu'il était responsable si son équipe n'avait pas remporté la coupe Grey. Même si de nombreux partisans voulaient "voir du sang ", les actions de l'entraineur nous ont calmés et nous ont redonné foi en notre équipe. De la même manière, dans la pratique de la pharmacie, nous devons tous être responsables de nos actions. Cette attitude contribue à assurer que nous gardons le respect de nos patients et des autres professionnels de la santé.

Petits gestes, grandes différences: Qu'il s'agisse de concéder un seul point dans un match de football ou de recommander une statine en prévention secondaire, ce sont de petits gestes qui peuvent faire de grandes différences à la longue. Nous devons tirer parti de chaque situation.

Attention aux détails: Qu'il s'agisse de compter le nombre de joueurs sur le terrain ou d'observer des tendances dans les taux de potassium, porter attention aux détails est vital.

Fierté, passion et inspiration : La Rider Nation peut faire une différence sur le moral des joueurs, avec ses chapeaux faits de vrais melons d'eau, ses chandails verts au nom des Roughriders, sa mascotte de 6 pieds, ses drapeaux et toutes ses figures peintes. Pour la pharmacie, c'est en montrant nos " vraies couleurs professionnelles " pour soutenir et mettre en valeur notre profession que nous pourrons faire une réelle différence pour la profession et pour nos patients!

En pensant à ce qui attend notre profession, je déborde de joie. Peut-être sommes-nous à l'aube d'une « Pharmacie Nation ». Je me demande ... quel fruit ou légume devrions-nous porter sur notre tête?

Jason Howorko, B.S.P., B. Sc., ACPR, est président et agent de liaison externe pour la $\mathrm{SCPH}$. 